\title{
Research Status of Impact on Concrete Structures
}

\author{
Zhang Chaofeng, Guan Zhongguo \\ Department of Bridge Engineering, Tongji University, Shanghai, China
}

\section{Email address:}

zcf199411@163.com (Zhang Chaofeng)

\section{To cite this article:}

Zhang Chaofeng, Guan Zhongguo. Research Status of Impact on Concrete Structures. Science Discovery. Vol. 9, No. 4, 2021 , pp. $194-199$. doi: 10.11648/j.sd.20210904.21

Received: June 26, 2021; Accepted: July 14, 2021; Published: July 15, 2021

\begin{abstract}
Concrete is currently the most widely used material in civil engineering structures. In addition to being subjected to dead loads and live loads during its service, it may also encounter accidental loads such as impacts and collisions. Previous research has shown that the forced characteristics of concrete structures under dynamic loads such as impacts and collisions are very different from those under static loads. In order to summarize the dynamic characteristics of concrete structures and measures to reduce its impact damage, this article firstly introduced the mechanical characteristics of concrete structures under impact loads, including the dynamic force characteristics of concrete structures under high-speed, low-speed impact loads and confining pressure; Then reviews the achievements and development of domestic and foreign scholars on concrete impact research in recent years: the research status of concrete dynamic constitutive structure is summarized from the aspects of the sensitivity of concrete strength strain rate and the dynamic constitutive model; the research status of impact resistance of concrete structure is carried out from the aspects of high-speed, low-speed impact load and measures to reduce its impact damage. Finally the development trend of concrete structure impact is summarized and prospected, which will provide some references for the subsequent research on concrete structure impact.
\end{abstract}

Keywords: Concrete, Accidental Load, Forced Characteristics, Impact Load, Dynamic Constitutive

\section{混凝土结构冲击的研究现状}

\section{张超峰，管仲国}

同济大学桥梁工程系, 上海, 中国

\section{邮箱}

zcf199411@163.com（张超峰）

摘要：混凝土是当前土木工程结构中使用最为广泛的材料，在其使用期间内除了受到恒荷载、活荷载的作用外，还可能 遇到冲击、碰撞等偶然荷载。已有研究表明混凝土结构在冲击、碰撞等动力荷载作用下的受力特征与静力荷载下有很大 不同。为了总结混凝土结构的动态受力特性以及减小其冲击损伤的措施，本文首先介绍了混凝土结构在冲击荷载作用下 的受力特性, 其中包括混凝土结构在高速、低速冲击荷载以及围压状态下的动态受力特性; 然后综述了近年来国内外学 者关于混凝土冲击研究的成果与进展：从混凝土强度应变率敏感性和其动态本构模型方面对混凝土动态本构的研究现状 进行了总结; 从高速、低速冲击荷载以及减小其冲击损伤措施方面对混凝土结构的抗冲击研究现状进行了总结; 最后总 结了混凝土结构冲击发展趋势, 并对其发展趋势进行了展望, 为之后开展混凝土结构冲击的研究提供部分参考。

关键词: 混凝土, 偶然荷载, 受力特性, 冲击荷载, 动态本构 


\section{1. 引言}

日常生活中，工程结构受到冲击的事故时常发生，而 这些事故轻则造成一定的经济损失, 重则造成人员伤亡。 一般情况下, 冲击可分为高速冲击与低速冲击两大类, 其 中高速冲击以爆炸为代表, 其中包括恐怖袭击, 煤气或天 然气爆炸, 子弹打靶等, 有数据统计表明, 与炸弹有关的 恐怖袭击活动在全球恐怖袭击活动总数中位列第二[1], 刘 琥等通过子弹打击防弹玻璃的试验获得了防弹玻璃的破 坏过程, 并验证了防弹玻璃侵彻毁伤及防护机理[2]; 而低 速冲击则以撞击为代表, 比如船舶与桥梁的碰撞、车辆与 公路栏杆的碰撞、地震作用下桥梁主梁与挡块的碰撞等, 在目前海域船舶通航量逐年增长的同时, 较多的跨海桥梁 受到轮船撞击的事故时常发生[3], 2008年的汶川地震发生 后, 通过震后调查发现大量梁式桥的板式橡胶支座和钢筋 混凝土挡块发生了碰撞, 最终导致桥梁破坏倒塌 [4,5]。高 速冲击与低速冲击二者之间并没有明确的划分标准, 一般 认为冲击块的质量较小、冲击速度较快、冲击时间较短, 使被冲击结构发生局部穿透破坏的冲击为高速冲击; 而冲 击块的质量较大、冲击速度较低、冲击时间较长, 使被冲 击结构发生整体破坏的冲击为低速冲击。混凝土是建筑结 构中使用最为广泛的材料之一, 已有的冲击事故也大多涉 及混凝土结构, 因此系统地研究冲击荷载作用下混凝土结 构的响应规律、损伤状态, 并采取相应的措施减小其损伤 具有十分重要的意义。

本文首先介绍了混凝土结构在冲击荷载作用下的受 力特性, 然后综述了混凝土动态本构研究现状以及混凝土 结构抗冲击研究现状, 最后总结了混凝土冲击的发展趋势, 并对其发展方向进行了展望。

\section{2. 混凝土结构在冲击荷载作用下的受力特性}

冲击一般为瞬时、高度非线性的复杂动力学问题, 混 凝土结构在冲击荷载作用下会形成大量裂缝, 但是由于冲 击荷载作用时间较短, 裂缝通常得不到较大的扩展, 因此 混凝土结构在动态荷载作用下的受力特性与静力荷载作 用下有很大差异, 大量学者对混凝土结构在冲击等动力荷 载作用下的受力特性进行了相关研究。

为研究混凝土结构在高速冲击荷载下的动力响应, 张 志刚等利用直径为 $100 \mathrm{~mm}$ 的分离式霍普金森压杆装置[6], 在较高的应变率条件下, 对混凝土结构进行了动态压缩试 验, 结果显示: 混凝土材料存在一定的应变率敏感性。姜 芳利用轻气炮装置对混凝土柱进行了冲击加载试验[7], 结 果表明: 柱子的应力-应变关系曲线表现出滞回特性、应 变率增强效应和波形弥散特性。赖建中等利用直径为 $74 \mathrm{~mm}$ 的分离式霍普金森压杆装置 [8], 对直径 $70 \mathrm{~mm}$, 高 度 $35 \mathrm{~mm}$ 的混凝土圆柱体试件进行了冲击压缩试验, 结果 发现其应力-应变曲线所包围的面积随应变率的增加也显 著增大, 同时还发现试件峰值应力、破坏应变也随应变率 的增加也增大。

为研究混凝土结构在低速冲击荷载作用下的动力响 应, 李静进行了混凝土短柱受轴向冲击的落锤试验 [9], 得
出结论: 短柱所受冲击力与冲击速度成线性正相关; 混凝 土短柱在轴向冲击荷载作用下会发生脆性破坏; 随着冲击 能量的增加, 短柱的破坏形式有一个从上而下逐步发展的 过程, 初始的冲击能量主要是通过混凝土短柱顶部的变形 甚至碎裂来耗散的。孟一通过落锤冲击试验对混凝土圆柱 体试件进行了研究 [10], 试验记录了锤头冲击力和试件纵 向应变时程曲线, 并利用高速摄像机记录下试件破坏的整 个过程。得出结论: 冲击力峰值、平均最大应力、应变率 均随着冲击速度的增加而增大, 而冲击时间则相反。

为研究混凝土结构在围压状态下的动力特性, 雷震等 利用落锤装置对“T”形截面钢管混凝土短柱进行了轴向冲 击试验[11]。结果表明: 短柱的冲击力峰值与壁厚成正比, 而坚向变形、冲击时间则相反; 钢管能够起到约束混凝土 的作用, 因此增加钢管壁厚可提高短柱的抗冲击能力。马 骐等利用落锤试验装置对 L 形截面钢管混凝土短柱进行了 轴向冲击试验[12], 结果表明: 冲击应力峰值、试件变形 均随着冲击高度的增加而增大。

以上学者的研究表明混凝土结构在冲击等动载条件 下的抗压强度确实高于静态抗压强度; 增加围压在一定程 度上可以提高混凝土的抗冲击能力。

\section{3. 混凝土动态本构研究现状}

\section{1. 混凝土强度应变率敏感性}

\subsection{1. 抗压强度应变率敏感性}

1917年, Abrams在试验中发现, 混凝土的抗压强度会 随着应变率的增加而增大 [13], 为了进一步验证应变率与 混凝土抗压强度的关系, 更多的学者开展了相应的研究。 如Bhargava等在1977年利用霍普金森压杆试验装置对素 混凝土试件进行了动态压缩试验 [14], 结果表明在动态荷 载下, 混凝土的抗压强度提高了1.45倍。随后, Bischoff 等对一圆柱体的素混凝土试件进行了单轴抗压试验 $[15,16]$, 结果表明: 在冲击荷载作用下, 混凝土材料的抗 压强度会增加 $0.5 \sim 0.6$ 倍, 并且混凝土材料的强度越高, 其 率增强效应越明显。胡功笠等使用直径为 $37 \mathrm{~mm}$ 的霍普金 森压杆试验装置对素混凝土试件进行了应变率在 $110 \mathrm{~s}^{-1} \sim$ $533 \mathrm{~s}^{-1}$ 之间的动态力学性能研究 [17], 结果发现: 随着加载 速率的增大, 试件的应力-应变曲线表现出由硬化向软化 过渡的趋向。Xiao等利用霍普金森压杆装置研究了素混凝 土的轴向冲击性能[18], 结果发现试件在高速冲击荷载下 的强度要高于静载, 且提高的比例随应变率的增加而增大。 张玉敏采用自行设计的试验装置并结合MTS液压伺服作 动器 [19], 对强度等级为 C25-C60的混凝土模制棱柱体试 件和 C30-C60的切割棱柱体试件分别进行了应变率在 $10^{-5} \mathrm{~s}^{-1} \sim 10^{-1} \mathrm{~s}^{-1}$ 之间的动态试验, 结果表明: 随着应变率的 增加, 混凝土的抗压强度也不断增大。

\subsection{2. 抗拉强度应变率敏感性}

一些学者对混凝土抗拉强度的应变率敏感性也进行 了相应的研究, 如Suaris等利用落锤试验机对混凝土梁进 行了冲击加载试验[20], 发现混凝土梁的弯曲抗拉强度表 
现出明显的应变率相关性。Klepaczko J R 等对干、湿混凝 土进行了动态抗拉强度试验 [21], 其准静态抗拉强度分别 是 $0.5 \mathrm{MPa}$ 和 $0.4 \mathrm{MPa}$, 给出了应变率直到 $10^{2} \mathrm{~s}^{-1}$ 的结果。 $\mathrm{Lu}$ 等利用分离式霍普金森压杆[22], 对混凝土试件进行了动 态䢃裂拉伸试验, 其试验装置如图1所示, 结果显示当加 载应变率超过 $10 \mathrm{~s}^{-1}$ 时, 试件的抗拉强度将随着应变率的增 加而增大。

运动方向 整形垫

图1 分离式霍普金森压杆的动态䢃裂试验装置。

\section{2. 混凝土动态本构模型研究进展}

混凝土的动态本构模型比较复杂, 近年来较为成熟的 模型为塑性模型和塑性损伤模型。塑性模型以热力学理论 为支撑, 在一定程度上能够得到一系列反映材料力学性能 的结果, 如弹性区域、失效条件、应变硬化效应等。塑性 损伤模型则以连续介质损伤理论为支撑, 能够考虑混凝土 内部的微裂纹在加、卸载过程中的扩展或闭合所导致的其 强度与刚度的变化。

\subsection{1. 塑性模型研究进展}

为研究应变速率对混凝土动态本构模型的影响, 肖诗 云等将应变率因子引入到传统Willam-Warnke三参数混凝 土本构模型中 [23], 建立了应变率相关性动态本构模型。 林臬等通过对混凝土材料进行双轴循环加载试验[24], 得 出了率型四参数弹塑性本构模型。L.Tashman等在Perzyna 本构模型基础上给出了一种粘塑性本构模型[25]。

通过以上学者的研究, 可以发现混凝土塑性本构模型 与应变率有关, 但是由于其不能考虑混凝土裂缝开裂与闭 合所造成的结构强度下降和提高的影响, 因此其应用范围 较为受限。

\subsection{2. 塑性损伤模型研究进展}

由于混凝土材料在冲击荷载下会发生损伤软化现象, 而塑性损伤模型由于考虑了混凝土裂缝的张开和闭合所 引起的混凝土强度和刚度的退化和增强, 因而更适合于混 凝土的动态力学分析。

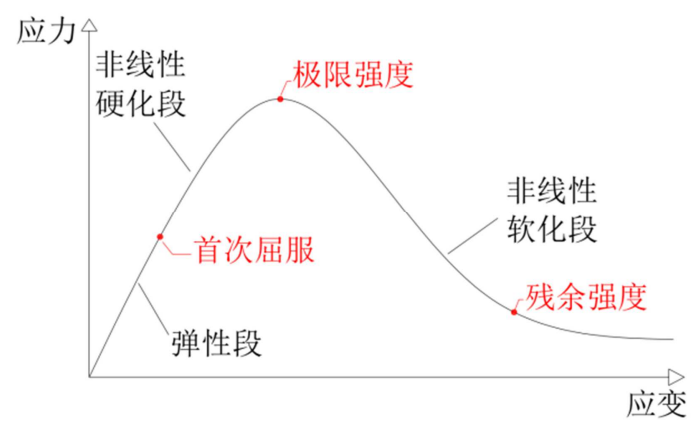

图2 混凝土三轴压缩的应力-应变曲线。
目前国外对于塑性损伤模型的研究较多, 如Qi等在 Faria模型的基础之上, 引入了黏性项和刚度阻尼项 [26], 得出了一种率型黏塑性损伤模型。Omidi等以Lubliner模型 为基础[27], 考虑损伤准则和黏塑性模型, 提出了率相关 黏塑性损伤本构模型。Holmquist等参考静水压力与体积应 变之间的关系 [28], 提出了 HJC混凝土本构模型, 其可以 分为三个部分即弹性段、过渡段和压实段。为了描述混凝 土材料在加载过程中的硬化和软化, Malvar等提出了混凝 土塑性损伤力学模型-K\&C模型[29]（如图2所示）, 该 模型能够反映出混凝土应力-应变关系在加载过程中的三 个阶段即弹性段、非线性硬化段和非线性软化段。

国内相关学者也对混凝土塑性损伤模型进行了相应 的研究, 如李杰和吴建营从混凝土基本损伤机理出发 [30,31], 得出了一种满足能量守恒和热力学定律的损伤型 本构模型。陈大年等以连续损伤理论为基础[32], 提出了 一种经验型混凝土率相关盖帽本构模型。周敉等对多种钢 筋混凝土梁模型进行了落锤冲击的数值模拟和对比分析 [33], 结果表明损伤模型和弹塑性帽盖模型能够更精确地 模拟裂缝发展和混凝土损伤变化。

以上学者的研究表明混凝土塑性损伤模型由于考虑 了混凝土微裂纹的发展和闭合, 能够考虑刚度、强度的退 化和提高, 因此更能适用于混凝土结构冲击的计算与分析。

\section{4. 混凝土结构抗冲击研究现状}

\section{1. 高速冲击状态下混凝土结构抗冲击研究进展}

一般认为应变速率在 $10 \sim 10^{6} \mathrm{~s}^{-1}$ 之间的冲击为高速冲 击, 目前高速冲击下混凝土结构的抗冲击特性主要是通 过分离式霍普金森压杆 (SHPB) 试验机（如图3所示) 完成的, 如 $\mathrm{Li}$ 等利用分离式霍普金森压杆试验装置对混 凝土试件进行了动态压缩试验[34], 结果发现: 当试验加 载的应变率超过 $100 \mathrm{~s}^{-1}$ 以后, 静水压力会对混凝土的动态 抗压强度产生增强的效应。施绍表等利用分离式霍普金 森压杆试验装置对强度为 $30 \mathrm{MPa}$ 的混凝土试件进行了单 轴冲击压缩试验[35], 得到了在大变形、高加载速率、高 静水压力条件下, 混凝土试件动态强度的变化。胡时胜 等利用改装的直径为 $74 \mathrm{~mm}$ 的变截面式霍普金森压杆试 验装置[36], 对圆柱体混凝土试件进行了冲击压缩试验, 结果表明: 混凝土材料具有应变率增强效应、损伤软化 效应。

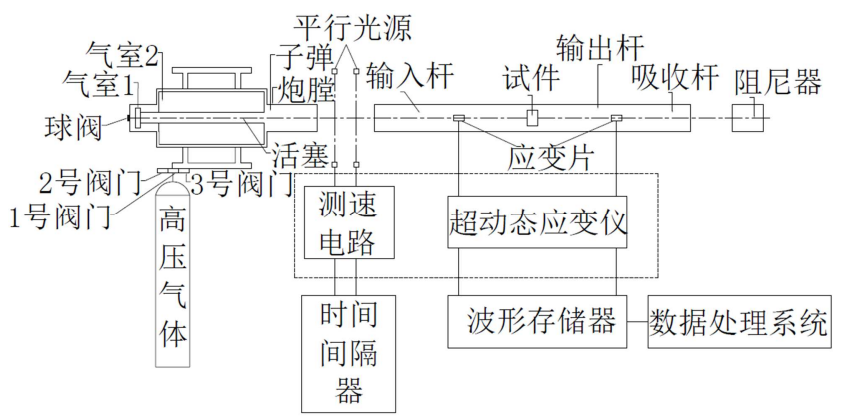

图3 霍普金森压杆试验。 


\section{2. 低速冲击状态下混凝土结构抗冲击研究进展}

一般认为应变速率在 $10 \mathrm{~s}^{-1}$ 以下的冲击为低速冲击, 目 前低速冲击下混凝土结构的抗冲击特性主要是通过落锤 试验机 (如图4所示) 和电液伺服试验机 (如图5所示) 完 成的。如Chen等利用落锤试验装置对钢筋混凝土梁开展了 冲击加载试验 [37], 试验中得到了锤头冲击力、试件加速度、 钢筋应变等时程曲线。黄涛利用落锤试验机对混凝土棱柱 体进行轴向冲击试验[38], 并利用超声仪检测冲击造成的 损伤程度,最后使用 $5000 \mathrm{kN}$ 压力机获得损伤后混凝土的应 力-应变曲线,得到损伤后混凝土力学参数的变化。间东明 等利用MTS液压伺服试验机完成了混凝土试件的单轴动 态拉伸试验[39], 并给出了体现应变率影响的经验计算公 式。

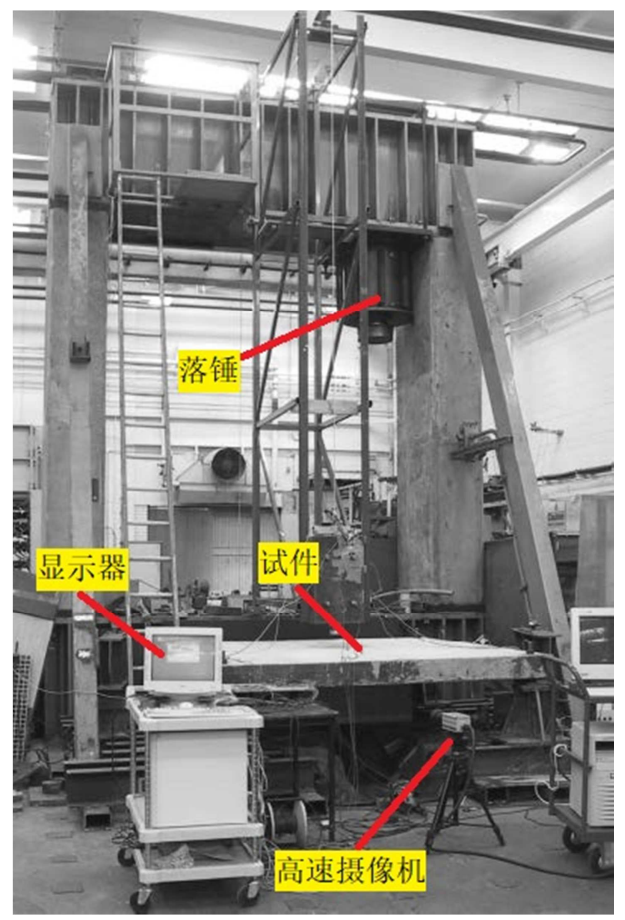

图4 落锤冲击试验设备。

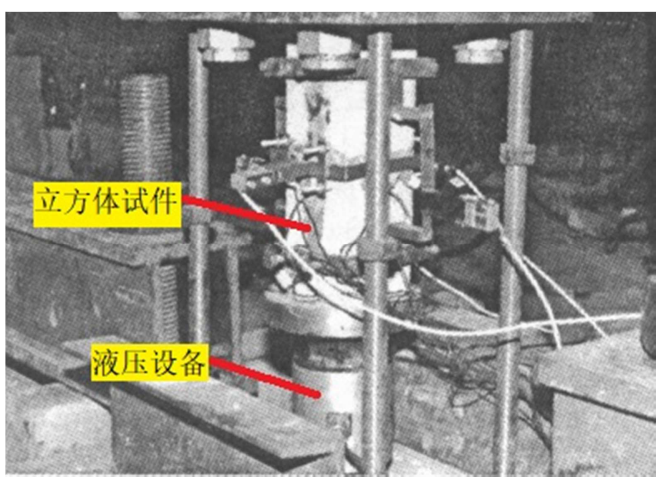

图5 电液伺服试验机。

\section{3. 减小混凝土结构冲击损伤措施研究进展}

为研究围压状态下混凝土结构的抗冲击性能, 李胜林 等利用霍普金森压杆装置对CFRP加固的混凝土柱进行轴
向冲击压缩试验[40], 得出碳纤维布加固可以提高混凝土 抗冲击性能的结论。李湘云对混凝土短柱进行了落锤冲击 试验[41], 得到了不同层数碳纤维包裹下混凝土短柱的破 坏模式和应变响应, 并且得出了落锤峰值力与短柱静承载 力之间的关系。梁家琦利用落锤试验装置[42], 通过改变 锤头初始动能分别对不同层数CFRP环向围束混凝土柱进 行了轴向冲击试验, 得出结论: 不同层数CFRP约束混凝 土试件的破坏形态相似, 并且在冲击荷载作用下, 层数越 多, 对混凝土的保护作用越强。马士宾等在混凝土试件中 添加聚丙烯纤维[43], 并对其抗冲击性能进行了试验研究, 得出结论: 试件添加聚丙烯纤维后的抗冲击韧性提高, 且 其抗裂能力与纤维掺量成正比; 试样厚度越大, 混凝土的 抗冲击强度越大。

陈庆丰等利用落锤试验装置对添加碳纤维的混凝土 试件进行冲击试验[44], 试验变量为碳纤维掺量和长度, 得出结论: 碳纤维能够增强混凝土强度, 限制裂缝的发展; 并且碳纤维掺量和长度均存在一个最优值。李亮等利用落 锤试验装置对添加钢纤维的混凝土配筋梁进行了冲击试 验[45]。结果表明: 添加钢纤维的配筋梁的韧性会增强; 梁的抗冲击能力会随着冲击荷载作用时间的增加而提高。

为研究施加缓冲层对混凝土柱破坏模式的影响, 孟一 对混凝土圆柱体试件进行落锤冲击试验研究 [10], 得出结 论: 在试件端部放置橡胶垫, 可以有效地减小柱体端部碰 撞损伤。李晓波通过建立提离式桩精细化有限元碰撞模型 也发现在桩端放置弹性缓冲垫能够减小桩端的碰撞损伤 [46]。

由以上学者的研究可以得出结论: 应变率对混凝土的 抗冲击性能影响显著; 围压状态、在试件内部添加纤维、 施加缓冲层均能提高混凝土结构的抗冲击性能。

\section{5. 混凝土结构冲击发展趋势及展望}

（1）目前对于混凝土在冲击荷载作用下的受力特性 研究主要是通过微观上的应变率来实现的, 而较少涉及到 混凝土结构受冲击后的宏观破环特征, 以及受冲击后的恢 复和再利用, 日后可加强后两种情况的研究。

（2）在混凝土的动态本构模型研究方面, 塑性损伤 模型是以塑性模型为基础, 考虑了损伤软化对材料强度、 刚度的影响, 能够较好地表达混凝土的动态本构关系。但 是已有的研究表明, 对于混凝土材料在动态压缩作用下的 本构模型研究较多, 而对其在动态拉伸荷载作用下的本构 模型研究较少, 因此需要深入研究混凝土材料的拉伸应力 -应变关系, 构建更加全面的混凝土动态本构模型。

(3) 以往的冲击试验中采用的冲击块大多是轻质量 的, 而且碰撞完成后二者之间几乎不再存有作用力, 这与 实际工程中的某些结构受力不符, 因为很多结构之间发生 碰撞之后会以高应力状态持续保持接触, 如可提离结构、 预制拼装结构在发生碰撞之后的复位过程中会一直以高 应力状态保持接触。因此以后的研究应该增加新型的冲击 试验装置, 探索混凝土结构在低速、高应力状态下的动态 受力特性。 


\section{6. 结语}

混凝土结构在冲击等动力荷载作用下的响应是一个 很复杂的过程, 不仅涉及到材料内部微裂纹的演化发展, 还涉及到材料应变率敏感性的影响。为了更深入地研究混 凝土结构在冲击等动载作用下的受力特性, 不仅需要建立 合适的混凝土动态本构模型, 还需要发明出较为先进的冲 击试验设备。研究混凝土结构冲击的最终目的是为了了解 混凝土材料在动载作用下的破坏特征和损伤机理, 并对易 受冲击的混凝土结构采取相应的措施减小其损伤, 在当前 混凝土结构受到冲击荷载的情况日益增多的状态下, 对混 凝土结构抗冲击性能的研究应该更加深入。

\section{参考文献}

[1] NCTC. A chronology of significant international terrorism for 2004[M]. U.S: National Counterterrorism Center, 2005: 83-84.

[2] 刘琥, 王胥, 项大林, 等. 防弹玻璃在子弹冲击下的数值 模拟研究, 导弹与航天运载技术 $[\mathrm{J}], 2017,46$ (5): 97-102.

[3] Ren Nianxin, Ou Jinping. A crashworthy device against ship-OWT collision and its protection effects on the tower of offshore wind farm[J]. China Ocean Engineering, 2009, 23(4): 594-602.

[4] 郭安宁, 郭增建. 从震源模式讨论 2008 年汶川 8 级地震的 回顾性预测 $[\mathrm{J}]$. 地震地质，2010,32（1）：115-126.

[5] 同济大学土木工程防灾国家重点实验室. 汶川地震震害 [M]. 上海: 同济大学出版社, 2008 .

[6] 张志刚, 孔大庆, 宫光明, 等. 高应变率下混凝土动态力 学性能SHPB实验 $[\mathrm{J}]$. 解放军理工大学学报(自然科学版), 2007, 8(06): 611-618.

[7] 姜芳. 钢筋混凝土材料动态力学性能的实验研究 [D]. 北京: 北京理工大学土木工程系, 2003：12-30.

[8] 赖建中, 孙伟, 焦楚杰. 生态型RPC材料的动态力学性能 [J]. 工业建筑，2004，34（12）：63-66.

[9] 李静. 钢管混凝土短柱轴向冲击的试验研究与仿真分析[D]. 太原理工大学, 2002 .

[10] 孟一. 冲击荷载作用下钢筋混凝土梁的试验及数值模拟研 究[D]. 湖南大学, 2012 .

[11] 雷震, 李召, 从地文, 等. 轴向冲击荷载下T形截面钢管混 凝土柱受力性能究 [J]. 建筑结构学报, 2017, 38 (1): 233-240.

[12] 马骐, 曾希, 雷震, 等. 轴向冲击荷载下 $L$ 形截面钢管混凝 土短柱受力性能 $[\mathrm{J}]$. 科学技术与工程, 2020, 20（8）: 3170-3178.

[13] Abrams D A. Effect of rate of application of load on the compressive strength of concerete. ASTM J, 1917, 17(Part II):364-377.
[14] Bhargava J, Rhenstrom A. Dynamic strength of polymer modified and fiber reinforced concretes[J]. Cement and Concrete Research, 1977,7(2):199-208.

[15] Bischoff P H, Perry S H. Compressive behavior of concrete at high-strain rates[J]. Materials and Structures, 1991, 24(144):425-450.

[16] Bischoff P H, Perry S H. Impact behavior of plain concrete loaded in uniaxial compression. Journal of Engineering Mechanics, 1995, 121(6): 685-693.

[17] 胡功笠, 刘荣忠, 齐爱东, 等. 混凝土材料的SHPB试验及 动态性能分析 $[\mathrm{J}]$. 南京理工大学学报, 2005, 29 (4): 420-424.

[18] Xiao Y, Shan J H, Zheng Q, Chen B S, Shen Y L. Experimental studies on concrete filled steel tubes under high strain rate loading. Journal of Materials in Civil Engineering, 2009, 21(10): 569-577.

[19] 张玉敏. 不同应变率下混凝土力学性能的试验研究 [D]. 北 京: 北京工业大学, 2012.

[20] Suaris W, Shah S P. Properties of Concrete Subjected to Impact. Journal of Structural Engineering, 1983, 109(7): 1727-1741.

[21] Klepaczko J R, Brara A. An experimental method for dynamic tensile testing of concrete by spalling[J]. International Journal of Impact Engineering, 2001, 25(4): 387-409.

[22] $\mathrm{Lu} \mathrm{Y,} \mathrm{Xu} \mathrm{K.} \mathrm{Modelling} \mathrm{of} \mathrm{dynamic} \mathrm{behaviour} \mathrm{of} \mathrm{concrete}$ materials under blast loading. International Journal of Solids and Structures, 2004, 41(1): 131-143.

[23] 肖诗云, 林㚖, 李宏男. 混凝土WW三参数率相关动态本构 模型 $[\mathrm{J}]$. 计算力学学报, 2004, 21(06): 641-646.

[24] 林臬, 间东明, 肖诗云等. 应变速率对混凝土特性及工程 结构地震响应的影响 [J]. 土木工程学报, 2005, 38(11): 1-8.

[25] Tashman L, Masad E, Little D, et al. A micro structure based viscoplastic model for asphalt concrete[J]. International Journal of Plasticity, 2005, 21(9):1659-1685.

[26] Qi Hu, Li Yungui, Lü Xilin. Practical elasto-plastic damage model for dynamic loading and nonlinear analysis of Koyna concrete dam[J]. Journal of Central South University, 2013,20(9): 2586-2592.

[27] Omidi O, Lotfi V. Continuum large cracking in a ratedependent plastic-damage model for cyclic-loaded concrete structures[J]. International Journal for Numerical and Analytical Methods in Geomechanics, 2013, 37(10): 1363-1390.

[28] Holmquist TJ, Johnson GR. A computational constitutive model for glass subjected to large strains, high strain rates, and high pressures[J]. Journal of Applied Mechanics-transactions of the Asme. 2011,78(5),1-9.

[29] Malvar LJ, Crawford JE, Wesevich JW, et al. A plasticity concrete material model for DYNA3D. International Journal of Impact Engineering. 1997, 19(9-10): 847-873.

[30] 李杰, 吴建营. 混凝土弹塑性损伤本构模型研究 I : 基本 公式[J]. 土木工程学报, 2005, 38(09): 14-20. 
[31] 吴建营, 李杰. 混凝土弹塑性损伤本构模型研究 II : 数值 计算和试验验证 $[\mathrm{J}]$. 土木工程学报, 2005, 38(09): 21-27.

[32] 陈大年, S.T.S.Al-Hassani, 尹志华, 等. 混凝土的冲击特 性描述 $[\mathrm{J}]$. 爆炸与冲击，2001，21（2）：89-97.

[33] 周敉, 温杰, 王超, 等. 碰撞荷载下钢筋混凝土动力本构 模型研究 $[J]$. 中国公路学报, 2017, 30(04): 59-71.

[34] Li Q M, Meng H. About the dynamic strength enhancement of concrete-like materials in a split Hopkinson pressure bar test. International Journal of Solids and Structures, 2003, 40(2): 343-360.

[35] 施绍雸, 王永忠, 王礼立. 国产 $\mathrm{C} 30$ 混凝土考虑率型微损伤 演化的改进Johnson-Cook强度模型 $[\mathrm{J}]$. 岩石力学与工程学 报，2006, 25(S1): 3250-3257.

[36] 胡时胜, 王道荣, 刘剑飞. 混凝土材料动态力学性能的实 验研究 [J]. 工程力学, 2001, 18(05): 115-118.

[37] Chen Y, May I M. Reinforced concrete members under drop-weight impacts. Structures and Buildings, 2009, 162(1): 45-56.

[38] 黄涛.冲击致损混凝土力学性能研究 [D]. 哈尔滨工业大学, 2013.

[39] 问东明, 林臬, 王哲, 等. 不同应变速率下混凝土直接拉伸 试验研究[J].土木工程学报, 2005, 38(06): 97-103.
[40] 李胜林, 刘殿书, 杨俊, 等. 碳纤维包裹下混凝土的动态 力学性能研究 $[\mathrm{J}]$. 公路交通科技, 2010, 27(04)：22-27.

[41] 李湘云. 碳纤维约束混凝土短柱抗冲击性能研究 [D]. 湖南 大学, 2011.

[42] 梁家琦. CFRP环向围束混凝土柱受低速冲击的试验研究 [D]. 长安大学, 2016.

[43] 马士宾, 徐文斌, 许艳伟, 等. 聚丙烯纤维高性能混凝土 抗冲击性能研究 [J]. 混凝土，2018，26（4）：26-29.

[44] 陈庆丰, 李少帅, 余龙泽, 等. 碳纤维混凝土抗冲击性能 研究 [J]. 河南工程学院学报(自然科学版), 2019, 31 (4): 28-31.

[45] 李亮, 姚云龙, 陈柏生, 等. 钢纤维混凝土配筋梁落锤冲 击试验研究 [J]. 混凝土，2019，41(04)：14-23.

[46] 李晓波. 地震作用下提离式RC桩桩端碰撞损伤分析与控制 [D]. 同济大学, 2016.

\section{作者简介}

张超峰（1994-）, 男, 河南人, 硕士生, 主要从事桥梁 抗震的研究。

管仲国（1976-）, 男, 辽宁人, 教授, 博士, 主要从事 桥梁工程教学与抗震研究。 